\title{
Experimental work over borehole filling material to reinforce characterization and model validation of Ground Heat Exchangers
}

\author{
A.J. Extremera-Jiménez ${ }^{1}$, D. Eliche-Quesada ${ }^{2}$, C. Guitérrez-Montes ${ }^{1}$ and F. Cruz-Peragón ${ }^{1}$ \\ ${ }^{1}$ Department of Mechanical and Mining Engineering, E.P.S. de Jaén, University of Jaén \\ ${ }^{2}$ Department of Chemical, Environmental and Materials, E.P.S. de Jaén, University of Jaén \\ Campus las Lagunillas s/n, 23071Jaén(Spain) \\ Phonenumber:+0034 953212367,e-mail: ajej0001@red.ujaen.es, cgmontes@ujaen.es, deliche@ujaen.es, fcruz@ujaen.es
}

\begin{abstract}
Ground Heat Exchangers (GHE) are part of Ground Source Heat Pumps (GSHP), employed as a renewable source in air conditioning systems. Models implemented are quite in tune to the real behaviour, enabling reliable designs and optimization, once several thermal properties of the surrounding soil and borehole are known. Since some values of those properties are evaluated from experiments, other ones are selected and approached from data base, leading sometimes to poor reproductions, due to the lack of their specific knowledge.

This work demonstrates the effectiveness of the experimental evaluation of the filling material properties in a vertical GHE, to guarantee more reliable values to model its thermal behaviour: moisture content, density, and both thermal capacity and conductivity. Experiments must be easy and fast to perform, because their values must be known previous to the GSHP design. In addition, an identification process over a numerical model has been developed. In order to check its consistency, results have been compared with those determined experimentally. As well as a high model accuracy, it has been demonstrated the importance of the presented assays to be included in the previous experimental work to the design step of a GSHP system, providing a valuable borehole characterization.
\end{abstract}

Key words. GSHP, GHE, borehole, thermal properties.

\section{Introduction}

A Ground Source Heat Pump (GSHP) is a relevant efficient system applied to air conditioning in buildings which involves high energy savings, leading to numerous environmental benefits [1]. The main difference with conventional systems is that the external heat exchanger device of the refrigerating machine interacts with the ground, instead of the surrounding air, being called Ground Heat Exchanger (GHE). Among several types, the vertical ones are quite efficient because the ground temperature remains mostly constant along depth up to $100-150 \mathrm{~m}$ deep.

For design and optimization purposes, several mathematical models have been developed, being currently very precise, at different levels [2]. But to tune these models, it is necessary the knowledge of several thermal properties. Most of them can be quite well adjusted from the called Infinite-Line Source model (ILS) and data collected from experimental tests: i) the undisturbed Ground Temperature Test (GTT) [3], where a fluid circulates in a laminar regime without heat input for a few minutes, and from which a mean constant value for the ground temperature $T_{S}(\mathrm{~K})$ is obtained; ii) the Thermal Response Test (TRT) [4] provides the inlet and outlet fluid temperatures to the GHE for a constant circulating flow along an experiment of twothree day duration, where a constant heat flow is injected.

Among all the characteristics, the most important one corresponds to the soil thermal conductivity $\lambda_{S}$ (W/m.K), being fortunately quite well fitted. The transient behaviour of the ground must be determined from the ground thermal capacity $C_{s}\left(\mathrm{MJ} / \mathrm{m}^{3} . \mathrm{K}\right)$ resulting in its thermal diffusivity $\alpha_{\mathrm{s}}\left(\mathrm{m}^{2} / \mathrm{s}\right)$. Its value is normally taken from tables, and does not represent a serious problem, because models show low sensitivities associated to this term. In relation with the borehole thermal behaviour, the ILS method can also provide the value of a property called borehole resistance $R_{b}$ (m.K/W) which involves the stationary behaviour of the grout, directly related to the borehole thermal conductivity $\lambda_{b}$ (W/m.K), although this last value is not really known. Errors associated to measuring, data acquisition and model, must be taken into account, having to assume a margin of error [5].

In addition, in recent years it has been demonstrated the importance of the intermittent behaviour of the GSHP, improving the device efficiency, when injecting the heat flow in the form of pulses [6]. It forces to take into account the borehole heat capacity $C_{b}\left(\mathrm{MJ} / \mathrm{m}^{3} . \mathrm{K}\right)$ (and in consequence its diffusivity $\alpha_{b}$ ), that the previous procedure is not capable to provide. The knowledge of both $\lambda_{b}$ and $C_{b}$ produces then a variable $R_{b}$, which is able to reproduce the system behaviour from heat pulses [7].

In this sense, numerical methods associated to GHE are reasonably attractive to identify this last term, as well as fitting other ones, such as $\lambda_{b}, \lambda_{s}, C_{s}, T_{s}, \ldots$ [8, 9]. In any case, these methods are also subject to sources of error, and could provide inconsistent results. In addition, the computation time is quite high, making unaffordable a practical procedure for a fast determination of properties to be used in a design step. Thus, it is necessary additional experimental work to provide more reliable properties associated to a friendly and reliable GHE model. 
The aim of this work is to demonstrate the convenience and effectiveness of adding easy experiments associated to the characterization of the filling material of a vertical GHE to those previous characterization steps (GTT, TRT, ILS, ...) of a GSHP design, obtaining fast results. For this purpose, grout thermal properties of a GHE, whose samples can be easily extracted from the borehole, have been evaluated from experiments. At the same time, a numerical model of that GHE has been developed, which has been subjected to a first approximation in the process of identification of parameters via Design of Experiments (DoE). Experimental results allow to discriminate the optimal range for this last process to select an effective set of thermal properties to tune the model.

\section{Material and Methods}

\section{A. GHE tested and data}

The GHE to be analysed (see scheme in Fig. 1) is located at University of Jaén (Spain), presenting a 150mm diameter and $130 \mathrm{~m}$ depth, in a single U-tube disposition of a $32 \mathrm{~mm}$ inner diameter PE100 pipe.

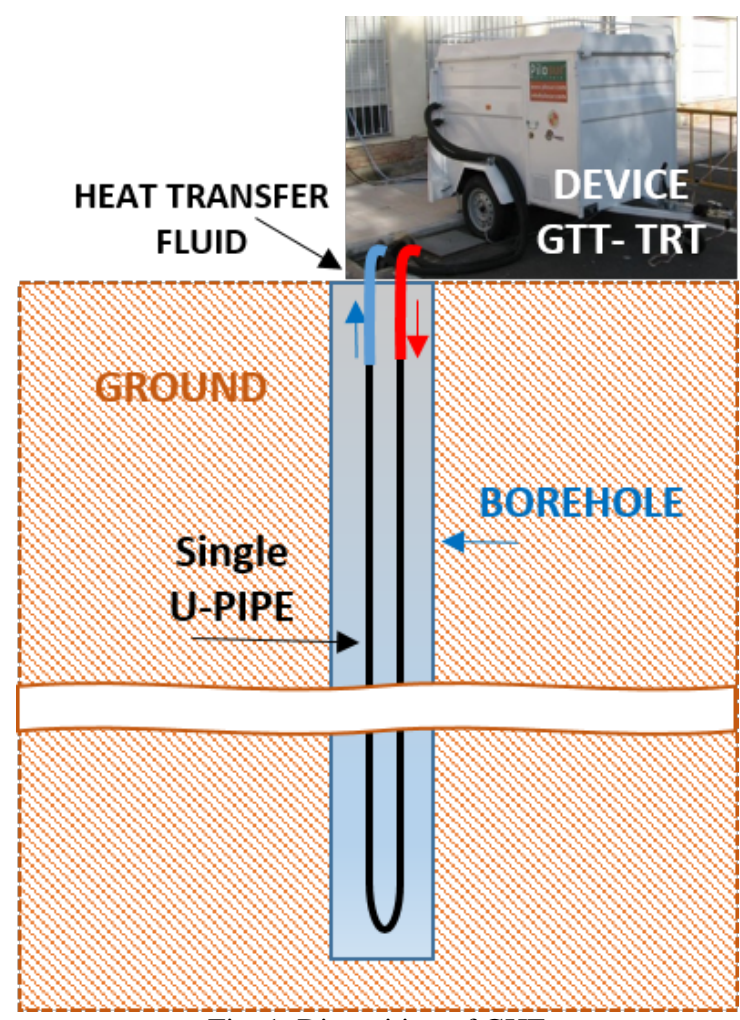

Fig. 1. Disposition of GHE

An overall procedure is shown in Fig, 2. A GTT was run first, obtaining a $T_{S}$ profile in depth, as well as its average value. In addition, a three-day duration TRT determines the inlet and outlet fluid temperatures, whose mean value $T_{f}$ allows providing $\lambda_{s}$ by a first step of the ILS method [10]. In a second step, $R_{b}$ (constant) is determined, providing in advance $C_{s}$ from other sources. A proper model associated to $R_{b}$ can also establish the borehole conductivity, $\lambda_{b}$ [11] (the opposite can also be done, that is, providing $\lambda_{b}$, from data base, modelling $R_{b}$ by [11] and then, establishing $C_{s}$. In any case, at least one these characteristic is not experimentally known for long-term simulation).

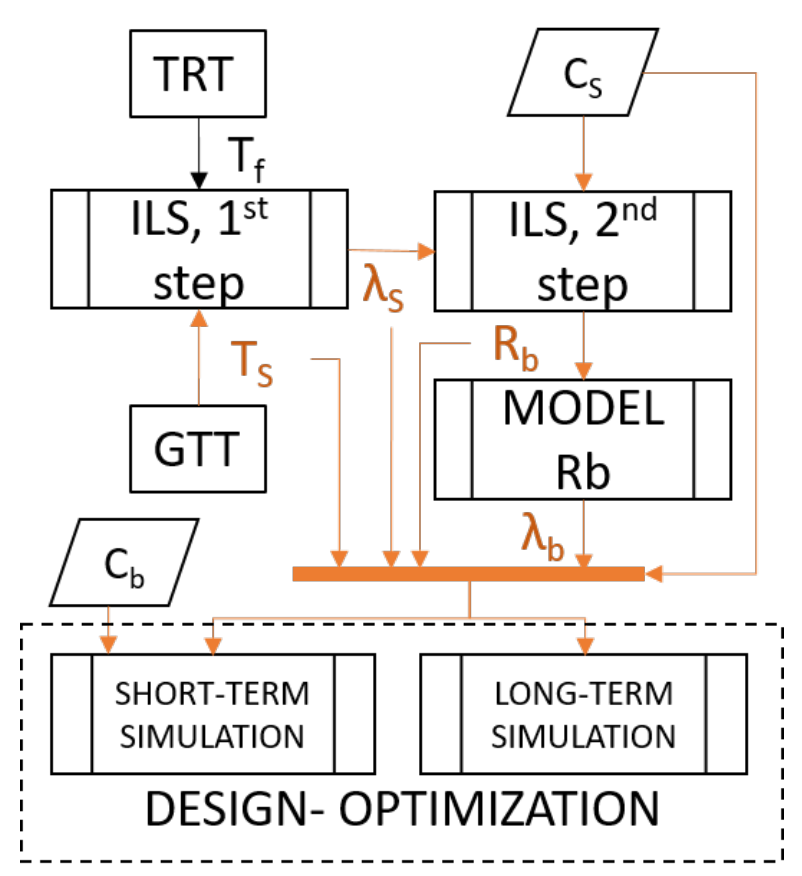

Fig. 2. Previous steps for thermal characterization of a vertical single GHE. Conventional procedure

These terms are then used by long-term models for a GSHP design or optimization stage. But for evaluating intermittent operation, short-term behaviour cannot be modelled only from those data, being necessary the additional knowledge of $C_{b}$ that must be searched independently. As a result, both models use two parameters not known from experiments (i.e. $\lambda_{b}$ and $C_{b}$ ).

\section{B. Numerical model and identification process}

Half of the described borehole (considering a symmetry condition, with adiabatic contour) was 3D modelled by a CFD commercial software by the Finite Volume Method (FVM) [12], including into the domain a $6 \mathrm{~m}$ diameter of the surrounding ground, as Fig. 3 shows.

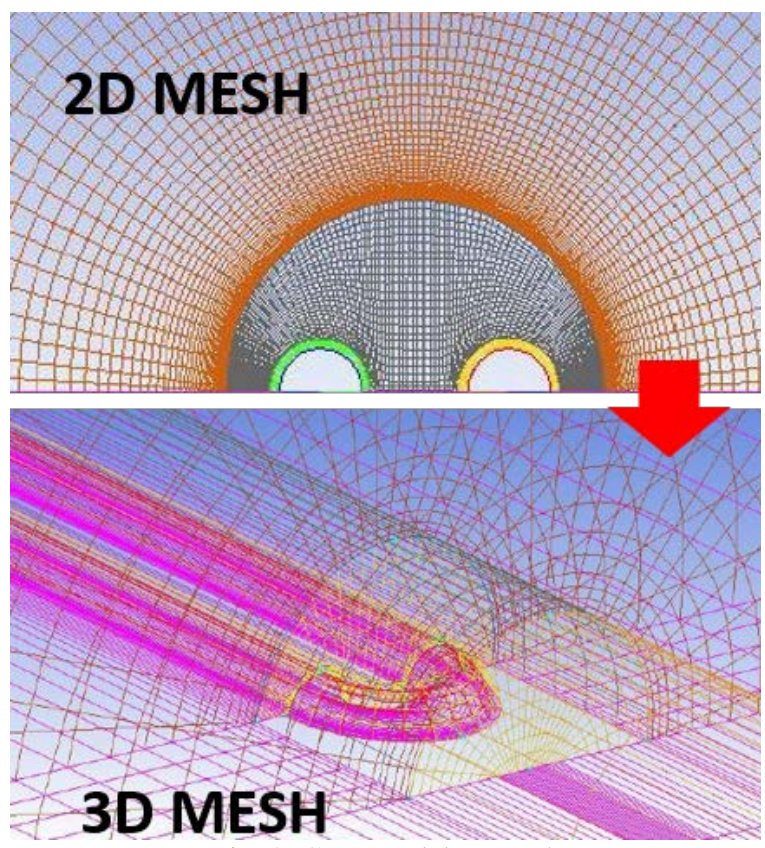

Fig. 3. GHE model network 
Thus, three-dimensional unsteady simulations of the problem have been conducted, where second-order accuracy in time and in space has been considered. In the outer surface of the control volume, it has been included as boundary condition $T_{s}$ (undisturbed ground temperature in depth from the GTT test), remaining constant along the TRT test. In addition, the overall system is initially at thermal equilibrium at the ground temperature, and the liquid at rest.

The model simulates the TRT experimented before, thus the inlet fluid temperature is set as input to it, to calculate the outlet fluid temperature, which is compared to that measured, and subsequently evaluate the model accuracy by means of the Root Mean Square Error (RMSE) between them.

To identify the suitable value for the borehole characteristics, a Design of Experiments (DoE) has been applied to the numerical model, searching the minimum RMSE [13]. For this purpose, the RMSE is fitted by a linear regression from a triangular array of binomial terms, representing, at least, a second order polynomial function of $R M S E \approx f\left(\lambda_{\mathrm{b}}, \mathrm{C}_{\mathrm{b}}\right)$. It allows to evaluate the shape of that $R S M E$, as response variable, depending on $k_{b}$ and $C_{b}$, as explanatory factors of the experiment (in this case, model),

This approach is built from a two-parameters, three-level, Full Factorial Design (FFD) of experiments, applied to those terms over a certain range determined initially from literature review. This design finds the path where to search that minimum, being necessary several steps. In this work, a first stage has been developed, in order to check if the optimal values that validate the model are within the margins given by the sample experiments.

\section{Experiments over borehole material}

Once the hole was filled, the following step consists of obtaining a sample of this material to be analyzed. In this case, an initial piece was extracted from the upper end of the borehole, and then, there was obtained a suitable section to perform the thermal conductivity analysis (see Fig. 4).

Further steps are as follows, where a precision balance Blauscal AH1200 was used for weighing materials along them:

1) The sample was dried first in a drying furnace Memmert (SNB 167 Model 100) to determine its equilibrium water content $X$ (kg w/kg d.b.), accordingly to ASTM D2216 standard [14].

2) Density $\rho_{b}$ : later, the dry density was determined following the ASTM C373-14 standard [15]. The sample was weighted twice: the first of them with dry mass $m_{d}$, and the second case, once they were saturated in air $m_{a}$. Later the saturated sample is weighted immersed in water, providing a value $m_{w}$. The dry density $\rho_{d}$ can be then calculated following the eq. (1), taking into account the water density $\rho_{w}$ $\left(\mathrm{kg} / \mathrm{m}^{3}\right)$ :

$$
\rho_{d}=\rho_{w} \cdot \frac{m_{d}}{m_{a}-m_{w}}
$$

As the equilibrium moisture is known, the material density $\rho_{b}$ with this water content can be then determined following eq. (2).

$$
\rho_{b}=\rho_{d} \cdot(\mathbf{1}+X)
$$

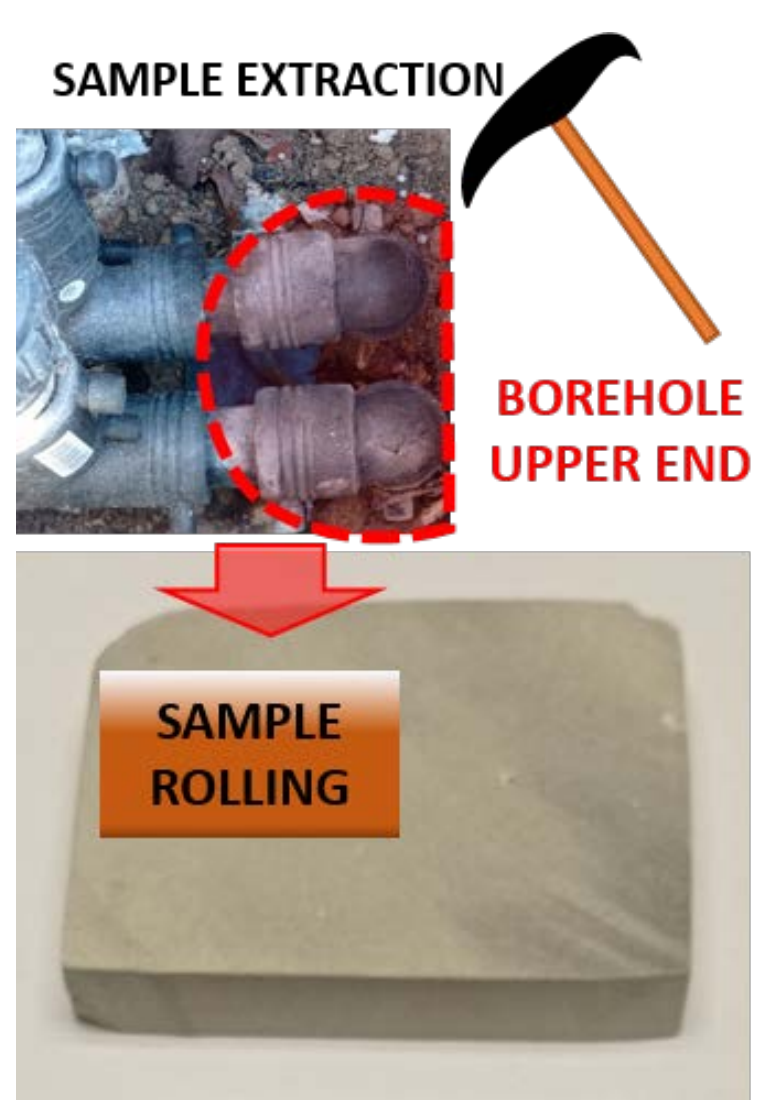

Fig. 4. Sample extraction and preparation

3) Thermal conductivity $\lambda_{b}$ : this characteristic has been determined with a guarded heat flow meter FOX-50 (TA Instruments), in accordance with the ISO 8302 standard [16], once adopted newly their equilibrium moisture content. As the rectangular shapes differ from those required by the device, it may produce discrepancies on experiments. Here appears a certain volume with air, presenting a thermal resistance $R_{A}$ in parallel with that corresponding to the sample $R_{S}$, as Fig. 5 shows. For an adequate value determination, the measured conductivity $\left(\lambda_{m}\right)$ provided by the equipment must be readapted following eq. (3), knowing the required section by the device $\left(S_{m}\right)$, the real one provided $\left(S_{b}\right)$ and the air conductivity $\left(\lambda_{a}\right)$.

$$
\lambda_{b}=\lambda_{m} \cdot \frac{s_{m}}{s_{b}}+\lambda_{a} \cdot \frac{s_{m}-s_{b}}{s_{b}}
$$

4) Heat capacity $C_{b}$ : this term is determined once the density $\rho_{b}\left(\mathrm{~kg} / \mathrm{m}^{3}\right)$, equilibrium moisture content $X$ (kg w/kg d.b.) and both the specific heat of wet and dry samples $c_{b}$ and $c_{d}$ (J/kg.K), respectively, are known, as eq. (4) shows.

$$
C_{b}=c_{b} \cdot \rho_{b}, \quad c_{b}=\frac{c_{d}+c_{w} \cdot X}{1+X}
$$



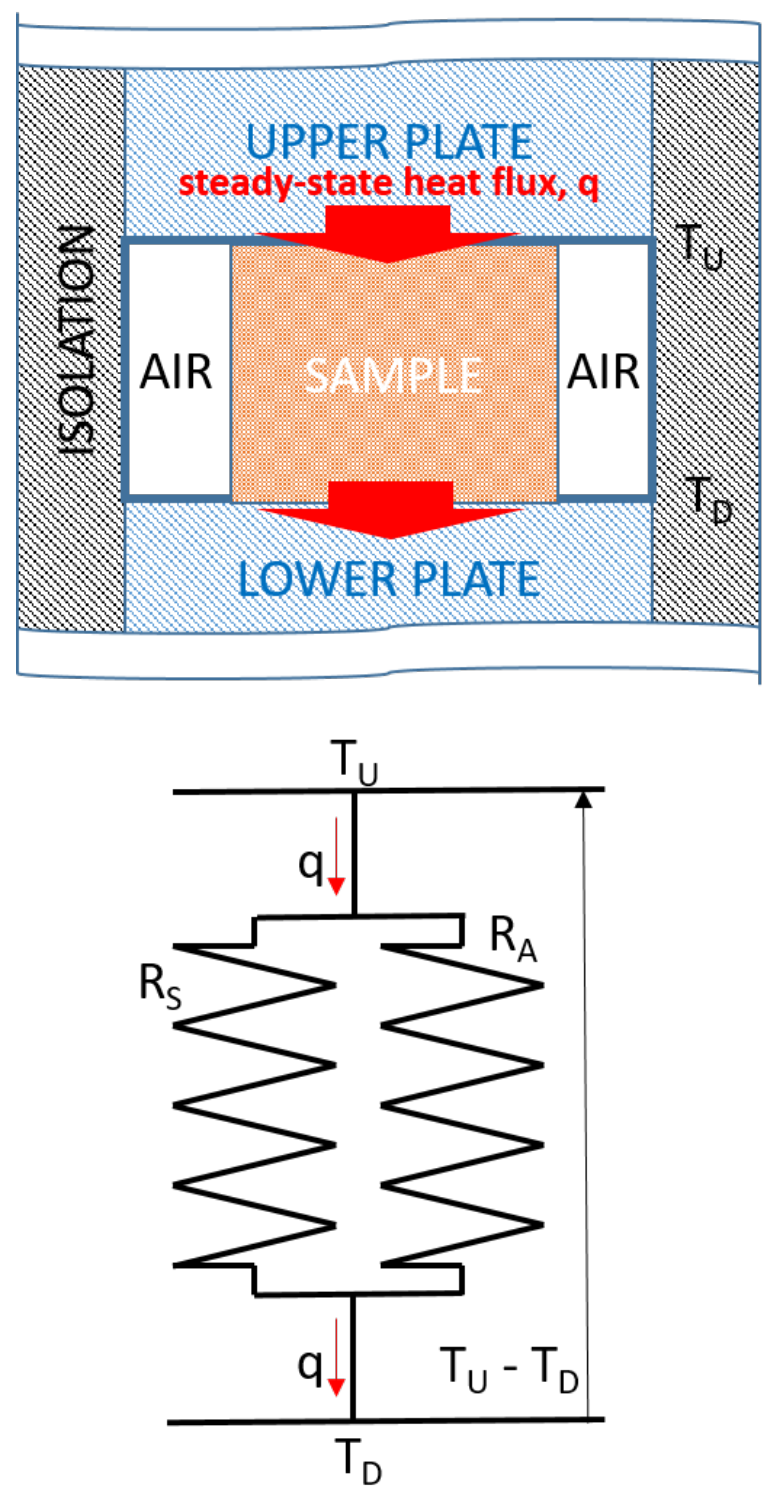

Fig. 5. Thermal resistance model at measurement device

The last property $c_{d}$ can be evaluated following the classical method of mixtures, accordingly to ASTM D4611 standard [17]. A heated dry sample at a temperature $T_{m i}$ is cooled with a mass of water $m_{w i}$, with a known initial temperature $T_{\text {wi }}$. After the thermal equilibrium is reached, the final temperature of the mixture $T_{m f}$ is used to determine this characteristic, following eq. (5).

$$
c_{d}=\frac{m_{w i}}{m_{d}} \cdot c_{w} \cdot \frac{T_{m f}-T_{w i}}{T_{m i}-T_{m f}}
$$

Previous to analyse the given results that eqs. (1) to (5) provide from assays, it has been evaluated the uncertainty $\omega_{R, j}$ associated to the determination of each parameter $R_{j}$ (such as $X, \rho_{b}, C_{b}$, and so on) by those formulae, from measuring $M_{i}$ (such as temperatures, masses, conductivity, surfaces, etc.). They depend on the their own uncertainties $\omega_{M, i}$, depicted in eq. (6) [18].

$$
\omega_{R, j}=\sqrt{\sum_{i}\left\{\left(\frac{\partial R_{j}}{\partial M_{i}}\right) \cdot \omega_{M, i}\right\}^{2}}
$$

A measurement uncertainty is frequently provided by the manufacturer of the corresponding device, incorporating irregularities at the sensor signal, the data acquisition system, and the calibration process (which are added in the same way as eq (6) shows). For the used equipment, the accuracies are equal to $0.005 \mathrm{~g}$ at the balance, $3 \%$ for the heat flow meter, and $0.1^{\circ} \mathrm{C}$ for the used thermistor. In addition, samples and experiments themselves induce errors that cause variabilities in results, thus it is important to repeat the tests several times.

\section{Results}

The numerical model has been run for the 9 combinations of values into their respective ranges for the input factors $\lambda_{b}$ and $C_{b}$ into the DoE. An example of the temperature field at an arbitrary moment for one of them can be seen in Fig. 6.

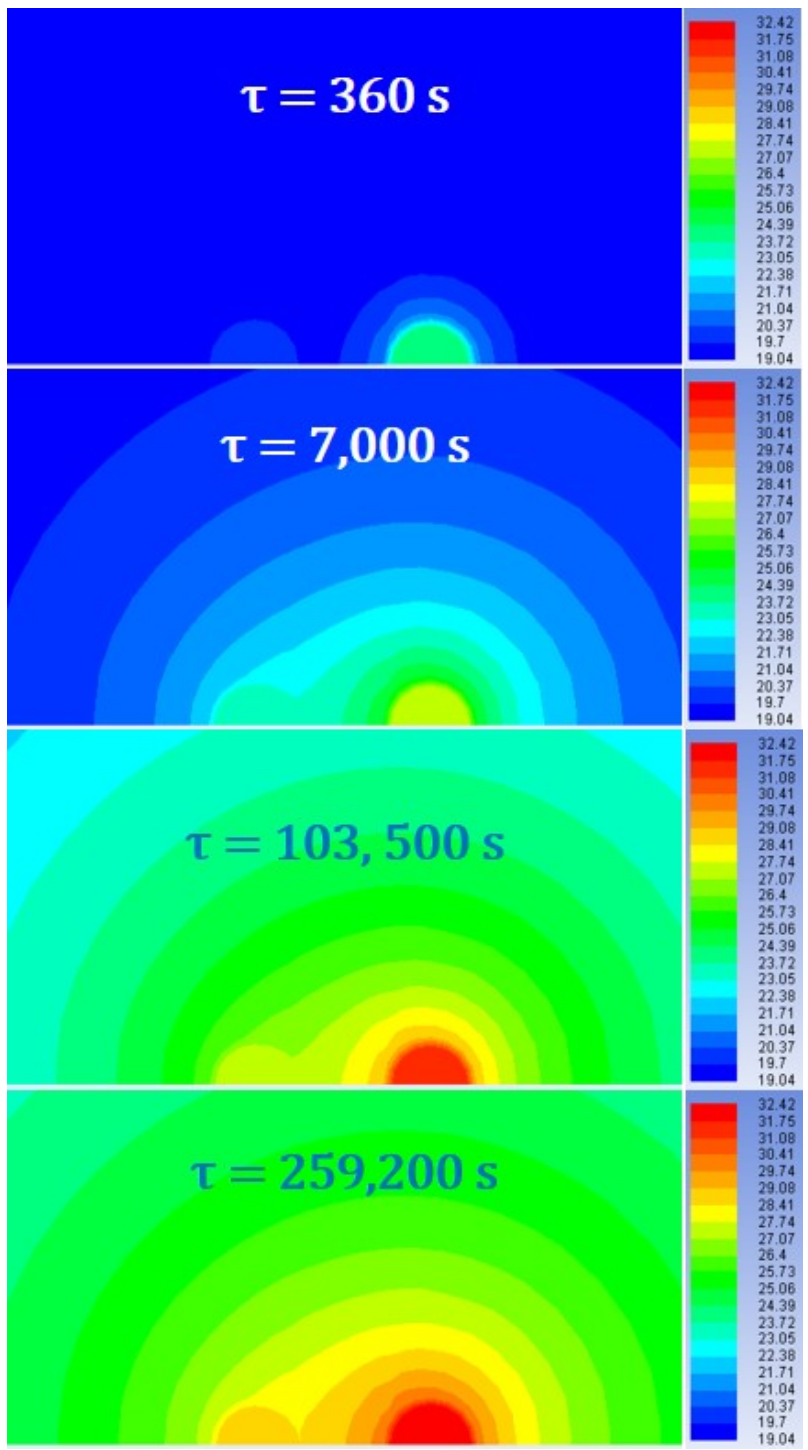

Fig. 6. Model results (temperature field in Celsius degrees) at borehole upper end along time of the TRT simulation

Obtaining the RMSE for these synthetic experiments, it has been approached the surface function for that indicator. RMSE surface results can be seen in Fig. 7 . 


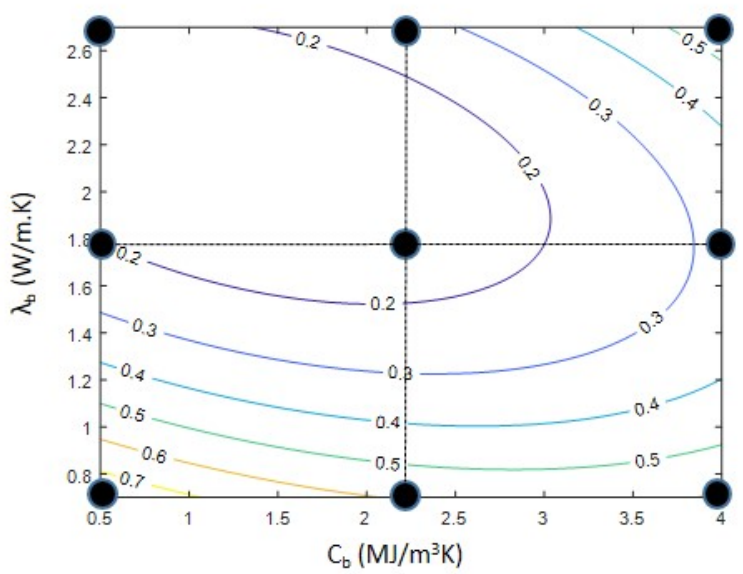

Fig. 7. RMSE surface of $T_{f}(\mathrm{~K})$ from DoE applied to the model

The advantage of this type of identification is that it is necessary a few number of runs of the model to point quickly to a little zone around the final solution. Nevertheless, results can provide local minimums, or for this case, extensive areas where minimum cannot be sensed (see Fig. 7). For a tighter approach, higher number of experiments are necessary, allowing upper orders at the RMSE surface approximation function. But it is quite important to select the most suitable zone to keep looking for the optimal solution, which must also present physical consistency. For this purpose, the help of additional data is essential.

In relation with experiments over the sample, they have been made in triplicate. As a result, uncertainties associated to measurements $\omega_{M, i}$ and equations $\omega_{R, j}$ for parameter determination are below $\pm 7 \%\left(6.5 \%\right.$ in $\lambda_{\mathrm{b}}, 5.95 \%$ in $C_{b}$, $0.5 \%$ in $X$, and $<0.1 \%$ for densities), being similar to those ranges observed in similar studies [18]. As they increase the initial deviations from experiments, the variability associated cannot assure a reliable unique solution for the borehole (depicted by their averages).

However, it is important to mention that the borehole is remarkably deep, and important changes in its thermal characteristics can appear along it, especially those related to the moisture content. Thus, it is more unfailing considering a certain range where physical consistency can be guaranteed but trying to assure those averages as determinant. These values are shown in Table I. Hence, ranges for $C_{b}$ and $\lambda_{b}$ from this table have been marked in Fig, 8 as a shaded shape over a zoom of Fig. 7 (as well as their mean values). As it can be seen, those ranges allow the selection of the best zone to search the final solution, increasing the number of experiments centered in that zone.

Table I. - Experiments results

\begin{tabular}{|l|c|c|}
\hline PARAMETER & Average & Range \\
\hline$X(\mathrm{~kg} \mathrm{w} / \mathrm{kg}$ d.b. $)$ & 0.041 & $0.036-0.048$ \\
\hline$\rho_{d}\left(\mathrm{~kg} / \mathrm{dm}^{3}\right)$ & 1.62 & $1.61-1.63$ \\
\hline$\rho_{b}\left(\mathrm{~kg} / \mathrm{dm}^{3}\right)$ & 1.68 & $1.67-1.68$ \\
\hline$\lambda_{b}(\mathrm{~W} / \mathrm{m} . \mathrm{K})$ & 1.65 & $1.46-1.83$ \\
\hline$C_{d}(\mathrm{~J} / \mathrm{kg} . \mathrm{K})$ & 558 & $381-681$ \\
\hline$C b(\mathrm{~J} / \mathrm{kg} . \mathrm{K})$ & 700 & $520-838$ \\
\hline$C_{b}\left(\mathrm{MJ} / \mathrm{m}^{3} . \mathrm{K}\right)$ & 1.18 & $0.88-1.41$ \\
\hline
\end{tabular}

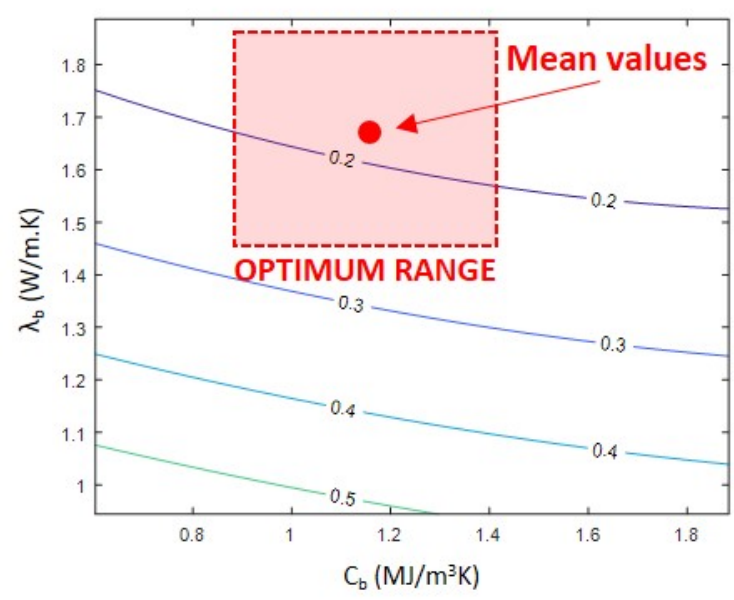

Fig. 8. Restriction of the area to search a consistent solution of identification process

By this way, it has been demonstrated, first, the usefulness of the presented experiment-based characterization, essential to provide consistent results from an identification process to tune a mathematical model of a vertical GHE.

Therefore, the experimental procedure is easy to perform in the previous steps of a GSHP design, as Fig. 9 shows.

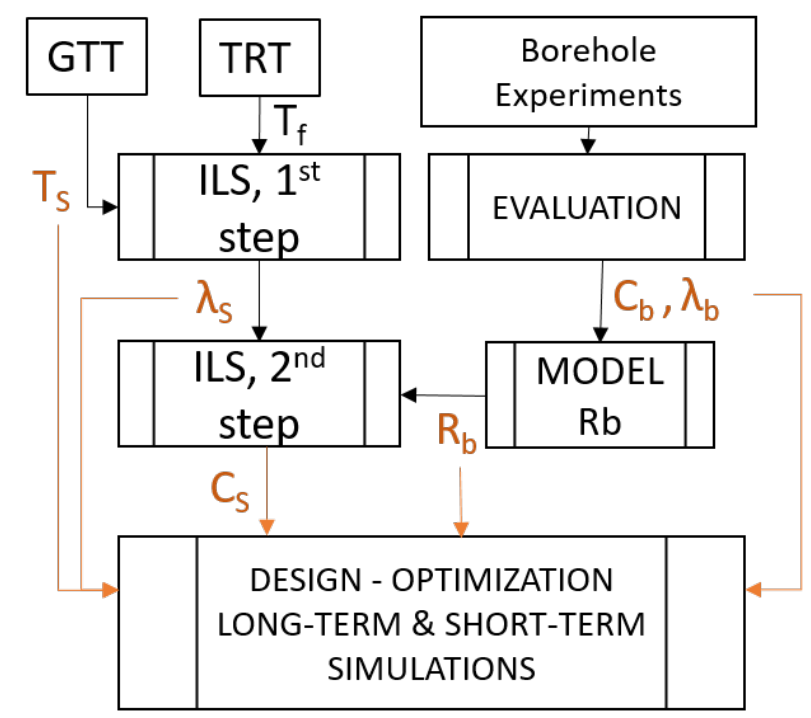

Fig. 9. Previous steps for thermal characterization of a vertical single GHE. Proposed procedure

Once a borehole is filled and dried, these samples can be taken on site before starting or immediately after finishing the tests over it (GTT, TRT), although maybe more appropriate molding them before that filling, with fresh mix. Later, results can be supplied at the same time of finishing the analysis of those tests, providing then a reliable characterization of a single borehole GHE. It serves as a starting point for a GSHP system design, even taking into account a short-term model for intermittent performance emulation.

A quite interesting advantage is that all the terms are experimentally derived, being included into the corresponding model (in both short-term and long-term ways). Neither $C_{S}$ nor $C_{b}$ must be inferred from tables or 
diagrams, as they can be identified following the scheme of Fig. 9. While in a traditional way, an estimated $C_{s}$ was used to determine $R_{b}$ by the ILS method, providing later $\lambda_{b}$ (see Fig. 2), now the opposite happens, where $R_{b}$ is given from $\lambda_{b}$ (experimentally), and later, the ILS method provides $C_{s}$. In any case, it is important to improve the experimental work associated to borehole samples, reducing the observed uncertainties in their thermo-physical characterization, and making possible to provide an effective solution for the set of terms $\lambda_{b}$ and $C_{b}$ (and consequently, $C_{s}$ ).

\section{Conclusions}

It has been demonstrated the usefulness of making experimental procedures applied to samples of the filling material of a borehole in a vertical GHE, due to the following reasons: i) by including this sample characterization within the previous ground characterization works (such as from GTT and TRT), it can be ensured that the values of all the parameters used by models are experimentally based (see Fig. 9), while conventionally, at least two of them must be supplied without this experimental basis (see Fig. 2). In any case, some of these experimental procedures must be improved; ii) when this characterization is applied to a model tuning, it helps to select a reliable range where to search the effective set of values for those considered parameters (see Figs. 7 and 8). It brings the initial values closer to the final solution, enabling a faster identification, as the number of model executions can be reduced.

\section{Acknowledgmens}

Authors thank to the project executed by the University of Jaén, with code 2439, for the financial support.

\section{References}

[1] M. Kharseh, L. Altorkmany, M. Al-Khawaj and F. Hassani, "Warming impact on energyuse of HVAC system in buildings of different thermal qualities and in different climates", Energy Conversion \& Management (2014), Vol. 81, pp. 106-111

[2] H. Yang, P. Cui and Z. Fang, "Vertical-borehole groundcoupled heat pumps: A review of models and systems", Applied Energy (2010), Vol. 87, pp. 16-27.

[3] R.A. Beier, "Vertical temperature profile in ground heat exchanger during in-situ test”, Renewable Energy (2011), Vol. 36, pp. 1578-1587.

[4] M. Li and A.C. Lai, Parameter estimation of in-situ thermal response tests for borehole ground heat exchangers", Intl. J. Heat Mass Transf. (2012), Vol. 55, pp. 2615-2624.
[5] F. Cruz-Peragón, P.J. Casanova-Peláez, R. López-García and J.M. Palomar-Carnicero, "Extending capabilities of Thermal Response Tests in vertical ground heat exchangers: An experiment-based local short-time temperature response factor", Applied Thermal Engineering (2020), Vol. 178, no. 115606

[6] A. Miyara, "Thermal performance investigation of several types of vertical ground heat exchangers with different operation mode”, Applied Thermal Engineering (2012), Vol. 33 pp. 167174.

[7] L. Lamarche, S. Kajl and B. Beauchamp, "A review of methods to evaluate borehole thermal resistances in geothermal heat-pump systems”, Geothermics (2010), Vol 39 (2), pp. 187200

[8] F. Bozzoli, G. Pagliarini, S. Rainieri and L. Schiavi, "Estimation of soil and grout thermal properties through a TSPEP (two-step parameter estimation procedure) applied to TRT (thermal response test) data”, Energy (2011), Vol 36(2), pp. 839-846

[9] J.C. Choi, S.R. Lee and D.S. Lee, "Numerical simulation of vertical ground heat exchangers: intermittent operation in unsaturated soil conditions”, Computer and Geotechnics (2011), Vol. 38, pp. 949-958

[10] P. Casanova-Peláez, J.M. Palomar-Carnicero, R. LópezGarcía and F. Cruz-Peragón, "Desarrollo de equipo para la realización de test de respuesta térmica del terreno (TRT) en instalaciones geotérmicas”, Dyna (2014), Vol. 89, pp. 316-324

[11] H. Zeng, N. Diao and Z. Fang, "Heat transfer analysis of boreholes in vertical groundheat exchangers", International Journal of Heat and Mass Transfer (2003), Vol. 46, pp. 44674481

[12] A.J. Extremera-Jiménez, Aproximación a la validación de modelo 3D de intercambiador de calor enterrado vertical (Master Thesis), Department of Mechanical and MiningEngineering, University of Jaén, Jaén (2019)

[13] C.F.J. Wu and M. Hamada, Experiments: Planning, Analysis, and Parameter Design Optimization, 2nd ed.; John Wiley \& Sons: New York (2000).

[14] ASTM D2216, Standard Test Methods for Laboratory Determination of Water (Moisture) Content of Soil and Rock by Mass, ASTM International, West Conshohocken, PA (2019)

[15] ASTM C373-14, Standard Test Method for Water Absorption, Bulk Density, Apparent Porosity, and Apparent Specific Gravity of Fired Whiteware Products, Ceramic Tiles, and Glass Tiles, ASTM International, West Conshohocken, PA (2014)

[16] ISO 8302, Thermal Insulation-Determination of SteadyState Thermal Resistance and Related Properties-Guarded Hot Plate Apparatus, International Standards Organization: Geneva, Switzerland (1991)

[17] ASTM D4611, Standard Test Method for Specific Heat of Rock and Soil, ASTM International, West Conshohocken, PA, (2016)

[18] M.H. Sharqawi, E.M. Mokheimer, M.A. Habib, H.M. Badr, S.A. Said and N.A. Al-Shayea, "Energy, exergy and uncertainty analyses of the thermal response test for a ground heat exchanger”, International Journal of Energy Research (2009), Vol. 33, pp. 582-592 